\title{
El derecho como forma de integración social según Habermas
}

\section{Law as a form of social integration according to Habermas}

\section{AYLTON BARBIERI DURÃO}

Doctor en Filosofía

Universidade Federal de Santa Catarina, Brasil ayltonbarbieri@gmail.com
Cómo citar este

artículo en APA:

Barbieri, A. (2021). El

derecho como forma de

integración social según

Habermas. Analecta

Política, 11(21), 299-314.

doi: http://dx.doi.

org/10.18566/apolit.

v11n21.a06

Fecha de recepción:

29.05.2021

Fecha de aceptación:

03.09.2021 


\section{Resumen}

Los problemas de integración en la sociedad compleja ocurren por causa de la división entre los sistemas político y económico, por un lado, que usan distintas formas de la racionalidad estratégica, basadas en el medio poder y dinero, respectivamente, y el mundo de la vida que usa la racionalidad comunicativa. En Teoría de la acción comunicativa, Habermas señala que la sociedad compleja lleva a un callejón sin salida en la medida en que los sistemas colonizan el mundo de la vida, pero, a partir de Facticidad y validez, muestra cómo los problemas de integración pueden ser resueltos por el derecho que usa las dos formas de racionalidad en virtud del doble sentido de su validez y, por eso, puede hacer la traducción entre el lenguaje comunicativo del mundo de la vida y el estratégico de los sistemas.

Palabras clave: Habermas, acción comunicativa, integración social, derecho, sistemas sociales.

\section{Abstract}

Integration problems in complex society occur because of the division between the political and economic systems, which use different forms of strategic rationality, based on middle power and money, respectively, and the world of life that uses communicative rationality. In Theory of Communicative Action, Habermas points out that complex society leads to a dead end as systems colonize the world of life. Nonetheless, from Facticity and Validity, he shows how problems of integration can be resolved by the law that uses the two forms of rationality by virtue of the double meaning of its validity. As a result, he can make the translation between the communicative language of the world of life and the strategic language of systems.

Keywords: Habermas, communicative action, social integration, law, social systems. 


\section{Introducción}

Habermas considera la sociedad moderna como compleja porque es dividida en sistemas sociales, entre los cuales se destacan la economía y la política, en que los agentes usan la racionalidad estratégica orientada hacia el éxito de la acción, mientras que, en el mundo de la vida, los agentes emplean el lenguaje ordinario y actúan orientados hacia el entendimiento según la racionalidad comunicativa. Además, hay diferencias entre la racionalidad estratégica empleada por los propios sistemas sociales, pues, en la economía, el éxito de la acción es medido por el medio dinero, mientras que, en la política, es sopesado por el medio poder. Pero si las personas y las instituciones sociales emplean distintas formas de racionalidad, entonces, es necesario explicar cómo es posible la integración social en la sociedad compleja.

Sin embargo, su punto de vista sobre los problemas de integración social en la sociedad compleja cambió a lo largo del tiempo en sus diferentes obras. Inicialmente, en Teoría de la acción comunicativa, Habermas consideró que el lenguaje ordinario, empleado en el mundo de la vida, constituye el último metalenguaje de los lenguajes formales de los sistemas sociales, por eso, su racionalidad comunicativa contribuye a la solución de los conflictos sistémicos y permite la integración social. No obstante, la débil fuerza de la racionalidad comunicativa del mundo de la vida raramente consigue guiar las acciones estratégicas de agentes en los sistemas sociales. Además, la economía y la política colonizan el mundo de la vida, lo que disminuye el espacio de la racionalidad comunicativa en el mundo de la vida y provoca la pérdida de este medio escaso. También el derecho fue entendido como un sistema social, especializado en la racionalidad estratégica y, por tanto, como contribuyendo para la colonización del mundo de la vida, por causa del incremento de la juridificación de las relaciones sociales. Por tanto, la economía, la política y el derecho constituían sistemas sociales que empleaban la racionalidad estratégica y colonizaban la racionalidad comunicativa del mundo de la vida, así como la pérdida de sentido de la racionalidad comunicativa del mundo de la vida aumenta los problemas de integración social en la sociedad compleja.

En las Tanner lectures, sin embargo, Habermas empezó a cambiar su perspectiva sobre el derecho porque observó su dupla pretensión de validez, por un lado, el derecho dispone de la facticidad que permite a los agentes, en especial los que actúan según la racionalidad estratégica de la economía y de la política, usar su autonomía privada y seguir la ley únicamente porque ella constituye un hecho social capaz de imponerse por coerción; por otro, el derecho permite que los agentes usen su autonomía pública y sigan las normas jurídicas por causa de su legitimidad según la racionalidad comunicativa. 
Este cambio de perspectiva respecto de la doble validez del derecho le permitió replantear su punto de vista sobre el papel del derecho en la integración en la sociedad compleja en Facticidad y validez a medida que el derecho puede servir para la traducción de la racionalidad comunicativa del mundo de la vida para la racionalidad estratégica de los sistemas sociales. En este sentido, el derecho actúa como un traductor universal entre el lenguaje ordinario del mundo de la vida y los lenguajes sistémicos entre sí. Por tanto, en el modelo planteado en Facticidad y validez, la sociedad compleja es presentada con tres niveles de acción social, mientras que la economía y la política, junto con otros sistemas, emplean la racionalidad estratégica y el mundo de la vida usa la racionalidad comunicativa, el derecho es desplazado a un papel intermediario entre los sistemas sociales y el mundo de la vida, porque los agentes pueden emplear simultáneamente las dos formas de racionalidad, lo que permite explicar la integración social.

\section{Los problemas de integración social en Teoría de la acción comunicativa}

La filosofía política y jurídica desarrollada por Habermas en la década de 1990 y publicada en Facticidad y validez (1992) y La inclusión del otro (1997) pretende dar una respuesta a los problemas antinomias resultantes de Teoría de la acción comunicativa (1981), además de mostrar cómo las exigentes condiciones de la ética discursiva, presentadas en Conciencia moral y acción comunicativa (1983), se pueden cumplir en las sociedades modernas.

Teoría de la acción comunicativa se escribió en la ambigua tradición de la primera generación de la escuela de Fráncfort, puesto que Adorno y Horkheimer insistían en que la racionalidad solo puede ser formal o instrumental, en la medida en que trasforma todo en medio y considera únicamente los fines relativos a la situación, según el cálculo costo-beneficio; por otro lado, Benjamin veía la posibilidad de una iluminación profana generalizada de las masas movidas por el arte de vanguardia engendrada por las nuevas tecnologías como la fotografía y el cine, mientras que Marcuse admitía la superación de la racionalidad instrumental en favor de una nueva ciencia y técnica capaces de establecer una relación armoniosa entre hombre y naturaleza, así como del hombre consigo mismo, retomando las ideas del joven Marx sobre el trabajo no alienado. 
Habermas jamás aceptó la posibilidad de una relación no instrumental del hombre con la naturaleza, hasta el punto de calificar los anhelos de Marcuse de una identidad en sentido hegeliano hombre/naturaleza de "la resurrección de la naturaleza caída, común a la mística judaica y protestante” (Habermas, 1973, p. 54). Él considera que la acción del hombre sobre la naturaleza, es decir, el trabajo con carácter casi trascendental, es siempre acción instrumental, pues al mismo tiempo que constituye la objetividad de los objetos fuera de nosotros, como un en-sí, se revela en su acto de aparición como un para-nosotros, y se transforma en la medida en que evolucionan las fuerzas productivas y la comprensión que los nuevos paradigmas tienen de la naturaleza. Habermas, sin embargo, observó que, además de la acción instrumental, existe otra forma de acción, la acción social, que rige las acciones entre los hombres. La acción social se divide en acción comunicativa y estratégica. La acción comunicativa ocurre cuando los hombres buscan el entendimiento mutuo, mientras que la acción estratégica se basa en el cálculo costo-beneficio sobre los efectos de la propia acción (Habermas, 1973, pp. 62-63).

Como la primera generación de la escuela de Fráncfort, Habermas absorbe el concepto de racionalización social de Max Weber en su teoría crítica de la sociedad, pero incorpora, además, las contribuciones de la sociología funcionalista de Parsons y Luhmann. Por consiguiente, describe la modernización social como un proceso de desencantamiento del mundo de la vida, en relación con el cual se desacoplan los sistemas funcionales. Habermas divide la modernización social en dos etapas. Entre los siglos XVI y XVIII, las esferas de valor se autonomizan en relación con el mundo de la vida, pero, a partir del siglo XVIII, los sistemas se constituyen en instituciones especializadas en coordinar la acción según el modelo de la racionalidad estratégica y regidos mediante regulación. El proceso de modernización social marca el cambio de la sociedad convencional a la posconvencional. La sociedad moderna posconvencional pierde el sentido holista representado por la identidad del ethos de la sociedad convencional. La sociedad moderna es compleja, fragmentada en sistemas organizados según racionalidades específicas. Eso tiene consecuencias para la estructura de la personalidad, pues, en la medida en que el individuo participa de múltiples sistemas, está obligado a cambiar su forma de acción de acuerdo con la lógica propia del sistema.

Los más destacados sistemas que se autonomizaron en relación con el mundo de la vida y que merecen mención por parte de Habermas, son el mercado y la política. El mercado es un sistema caracterizado por la racionalidad estratégica, que solo considera el cálculo psicológico de placer y sufrimiento implicado en el binomio costo-beneficio de la acción. El dinero funciona como medio de intercambio entre los agentes económicos. La política también es un sistema social 
fundado en la acción estratégica, cuya moneda de cambio entre los agentes sociales, sin embargo, es el poder. En la sociedad moderna, esos dos sistemas entran en competencia directa, y ofrecen dos alternativas para el Estado de derecho. Se puede plantear el modelo de Estado de derecho que deja al mercado la capacidad de autorregulación, libre de las intervenciones del sistema político; se amplía al máximo la esfera privada de los agentes económicos y se reduce al mínimo la esfera pública de la política. Por otro lado, las crisis continuas de superproducción del mercado y el subempleo de los factores económicos, el trabajo y el capital conducen al punto de vista opuesto de que el mercado necesita regulación política en el sentido de generar una distribución más equitativa de la riqueza. La modernidad se escindió entre el estado liberal y el estado de bienestar social. Habermas considera, no obstante, que esta antinomia en torno al concepto de Estado moderno no agota el problema de la complejidad social, porque los sistemas basados en la acción estratégica tienden a ampliarse continuamente, e invadir el ámbito del mundo de la vida, a lo cual denomina colonización del mundo de la vida $(\mathrm{Ha}-$ bermas, 1995 , v. 2, p. 470). Esa nueva antinomia es más grave que la anterior, puesto que en el mundo de la vida se preservan las relaciones de solidaridad y entendimiento mutuo de la acción comunicativa. La colonización del mundo de la vida representa la expansión de la racionalidad estratégica de los sistemas de acción y el encogimiento del dominio de la acción comunicativa. La esfera del mundo de la vida está cada vez más sometida al medio dinero y al medio poder. Habermas entiende que es alarmante tanto la alternativa liberal de la regulación social por las reglas del mercado como la intervención social promovida por el estado de bienestar para compensar las desigualdades sociales y económicas. El estado de bienestar, para cumplir su función, desarrolla una enorme burocracia que transforma a los ciudadanos en clientes de la política de asistencia social e implica la pérdida de la capacidad de participación política. Con la clientelización de la ciudadanía, los beneficios del estado de bienestar se pierden, incluso, porque los grupos presuntamente beneficiados se tornan marginados por el proceso económico y necesitan todavía más la burocracia estatal, en un círculo vicioso.

Este modelo sociológico desarrollado por Habermas en Teoría de la acción comunicativa, que intenta reconstruir el materialismo histórico con los medios de la teoría sistémica, presenta una imagen de la sociedad fragmentada en sistemas que se autonomizaron del mundo de la vida cada vez más impotente para hacer frente a los problemas de integración social. Los sistemas sirven para coordinar la acción, usando la racionalidad estratégica; pero, ni el mercado, con su pretensión de convertir la lógica del medio dinero en moneda de cambio de las relaciones sociales, puede generar la solidaridad necesaria para la convivencia entre los hombres, ni tampoco las compensaciones del estado de bienestar pueden rescatar una vida 
no malograda con el recurso del poder. El mundo de la vida, que en sociedades tradicionales cumplía la función de integración social, se vuelve debilitado por la autonomización de los sistemas, la lógica de la acción comunicativa tiene poco prestigio al lado de los sistemas funcionales, y al final se sustituye la coordinación de la acción por medio del entendimiento mutuo del mundo de la vida por la coordinación a través del dinero y del poder.

Con la pérdida del ethos tradicional resultante de la modernización sistémica, las sociedades modernas tienen grandes problemas de integración social. Habermas intenta explicar la integración social apelando al modelo de los discursos racionales, que serían formas especializadas de la acción comunicativa. Con la modernización social, también la acción comunicativa se torna reflexiva hasta el punto de que los actores disponen de la capacidad de poner en tela de juicio los valores tradicionales del mundo de la vida. Los discursos son procedimientos que se autonomizan del mundo de la vida, pero que reproducen de forma reflexiva la búsqueda cooperativa de la verdad, de la rectitud y de la sinceridad, que son desempeñadas ordinariamente por los actores en la acción comunicativa. Sin embargo, los discursos contribuyen ambiguamente a la integración social. En primer lugar, porque, aun cuando están relativamente instituidos, como en la comunidad científica o en los parlamentos, no poseen la fuerza institucional para obligar a los sistemas funcionales, con su integración funcional por medio del dinero o del poder; pero, en segundo lugar, los discursos generan otros problemas para la propia integración social, porque en la medida en que posibilitan que los actores sociales critiquen los valores básicos de la sociedad, destruyen la aceptación ingenua de esos actores y hacen posible seguir reglas de acción fundadas en la reflexión. Como consecuencia, las visiones de mundo y concepciones de vida buena se pluralizan, es decir, los discursos solo pueden mantener la integración social al precio de tornarla reflexiva y socavar las condiciones de la solidaridad (Habermas, 1994, p. 55).

Con el marco propuesto por la teoría crítica de la sociedad, el derecho no dispone de medios para superar las antinomias entre estado liberal y estado de bienestar. Habermas, profundamente influido por la sociología del derecho de Marx, Weber, Parsons y Luhmann, entiende el derecho como otro sistema social, regido por la racionalidad estratégica, y contribuyendo con el mercado y la política a la colonización del mundo de la vida, fenómeno descrito como juridificación de las relaciones sociales (Habermas, 1995, v. 2, p. 522). 


\section{El cambio de perspectiva sobre el papel del derecho para la integración social en las Tanner lectures}

En el artículo "Derecho y moral", publicado en las Tanner lectures en 1986 e incorporado posteriormente como apéndice a Facticidad y validez, comienza a modificar la concepción sobre el derecho y el Estado democrático de derecho de acuerdo con los términos de su investigación de la década de 1990. En primer lugar, polemiza con las críticas que Weber dirige contra la moralización del derecho. Para Weber, la legitimidad del derecho proviene de la fe en la dominación legal que tienen los ciudadanos. Es decir, los ciudadanos confían que el derecho sea legítimo a partir de tres razones: a) el sistema jurídico es completo y consistente, de modo que puede incluir todas las leyes necesarias para regular las acciones entre los hombres, leyes que no entran en conflicto entre sí; b) las leyes son formales y abstractas, lo que implica la igualdad de todos ante la ley, y c) el derecho puede ser aplicado como un simple cálculo en el cual el órgano judicial tipifica el caso e impone la pena, garantizando la vinculación del juez a la legislación (Habermas, 1994, p. 544). Pero este modelo liberal del derecho se pone justamente en tela de juicio con el advenimiento del estado de bienestar, pues se hace evidente que la igualdad formal ante la ley posibilita una enorme desigualdad material. Para generar una igualdad real entre los ciudadanos, el derecho sufre innumerables modificaciones en su interpretación por el estado social. El sistema jurídico pierde la coherencia y completud y pasa a ser comprendido como un conjunto repleto de antinomias y lagunas, cuya aplicación, por los jueces, depende de la jerarquización de las normas entre principios y reglas, puesto que las reglas son de aplicación inmediata y su conflicto implica la supresión de una de ellas, mientras que los principios son abstractos y permiten interpretación en el caso concreto. Por eso, la colisión de principios exige la ponderación sobre cuál debe prevalecer, aunque ellos permanezcan en el sistema jurídico y pueden predominar en otro caso, así como posibilitan llenar las lagunas por medio de la interpretación; las leyes dejan de ser formales y abstractas, y pasan a considerar las circunstancias y los grupos de intereses; por fin, la aplicación del derecho no resulta de un simple cálculo, sino de la interpretación de los jueces, y por tanto en la autonomización del órgano judicial en relación con la legislación. Weber entiende estos cambios como moralización del derecho, cuya destrucción del formalismo implica la eliminación de la propia confianza en su legitimidad (Habermas, 1994, p. 545). Habermas piensa que, a pesar de las diferencias entre derecho y moral, la legitimidad del derecho debe seguir el proceso discursivo análogo al discurso de fundamentación de las normas 
morales. En la segunda parte de su artículo, cuestiona la sociología del derecho de Luhmann, que defiende la tesis de la autonomía sistémica del derecho. Para Luhmann, el derecho es un sistema cerrado que produce autopoiéticamente las leyes; Habermas argumenta que la idea de Estado democrático de derecho depende de un concepto de derecho entrelazado con la política y la moral.

El derecho civil alemán empieza con la discusión sobre la legitimidad del derecho subjetivo introducida por Savigny y Puchta, quienes lo fundamentan en la noción de autonomía de la persona moral, de origen kantiano. Sin embargo, al ser socavada la base de fundamentación moral del derecho subjetivo, solo le quedó al positivismo jurídico una explicación del derecho a partir del utilitarismo de Windscheid, del voluntarismo del legislador según Austin o del positivismo lógico de Kelsen. Para Kelsen, el derecho subjetivo es una autorización positiva del derecho objetivo para preservar la esfera privada. El derecho puede ser deducido por el sistema jurídico a partir de una norma fundamental, apartándose de la moral y de la política, porque entiende que la actividad de producción y aplicación del derecho debe hacerse por un sistema jurídico especializado (Habermas, 1994, pp. 113-114). Al insistir en el entrelazamiento entre derecho, moral y política, Habermas pretende rescatar, en contra de los defensores del derecho subjetivo que buscan un fundamento moral para el derecho, el momento de instrumentalidad del derecho, pues es el resultado de la producción del legislador que dispone de la capacidad de actuar discrecionalmente. Por otro lado, desea recuperar el momento de legitimidad presente aún en el derecho subjetivo, aunque arrinconado por la evolución del derecho positivo. Un derecho autónomo en relación con la moral y la política, o aparece como el resultado de la voluntad arbitraria del legislador (Austin), o como un deber-ser empíricamente obligatorio que se impone a los actores (Kelsen) (Habermas, 1994, pp. 583-584).

A pesar de constatar Habermas, en "Derecho y moral", la doble base de validez del derecho, ya que puede ser impuesto instrumentalmente o fundamentado legítimamente, aún no emprende su reconstrucción de la filosofía kantiana del derecho y del concepto de legalidad en los términos en que lo hará en Facticidad y validez. Habermas argumenta que los dos momentos de validez del derecho: la instrumentalidad y la legitimidad, pueden ser distinguidos, como lo demuestra la evolución del derecho civil alemán, con la división entre derecho subjetivo, fundado moralmente, y derecho objetivo, deducido deontológicamente del orden legal; no obstante, la doble validez jurídica ya se podía encontrar mucho antes en el derecho natural racional de los siglos XVI y XVII, y este rasgo sirvió también para marcar la dicotomía entre los autores del periodo que acentuaban uno u otro polo. Hobbes, a partir del egoísmo racional del individuo, concluye que 
todo derecho es positivo e instrumental, porque el contrato social, que instituye la sociedad civil, exige que los individuos renuncien a sus derechos naturales en provecho de uno de los miembros, el soberano, que dispone del poder absoluto (Habermas, 1994, p. 590). Kant, por otro lado, reacciona a la instrumentalización del derecho y recurre al único derecho innato del hombre: la libertad. Él obtiene el concepto de derecho y el principio del derecho a partir de la reducción del principio moral expresado por el imperativo categórico. Por eso, el hombre posee derechos naturales fundamentados moralmente en el estado de naturaleza y tan solo debe realizarlos en el estado social. El derecho natural y la moral se entrelazan hasta el punto de que no se puede distinguir entre ellos, excepto por medio de la teoría de los dos reinos. Porque el derecho estricto puede imponer por la coerción una moral que solo puede aceptarse por motivación; sin embargo, a lo largo de la evolución de la Historia, marcada por el conflicto de la sociable insociabilidad, la providencia posibilita que la humanidad se acerque al reino de la moralidad, después de acostumbrarse a la imposición de la ley. Como queda comprobado por la oposición entre res publica noumenon y res publica phaenomenon (Habermas, 1994, pp. 590-591).

El entrelazamiento entre derecho, política y moral es necesario para superar la dicotomía del modo de validez del derecho que aparece tanto en el derecho natural racional como en el derecho civil alemán. El derecho no puede perder su carácter instrumental, lo que ocurre con la aproximación excesiva entre derecho y moral, so pena de devaluar el papel de la voluntad del legislador; pero tampoco reducirse a pura instrumentalidad, haciéndose simple resultado de la voluntad arbitraria, como sucede cuando se acomoda en exceso a la política. Sin embargo, la ausencia de reflexión en torno al concepto kantiano de legalidad no permite a Habermas, en este artículo, explicar adecuadamente el doble sentido de la validez del derecho dividida entre la facticidad de la imposición del aspecto coercitivo y la legitimidad proveniente de la aceptación de razones y argumentos que simultáneamente posibilita la formación de la voluntad.

\section{El papel del derecho como forma de integración social en Facticidad y validez}

Es solo en Facticidad y validez en que Habermas emprende la reconstrucción del concepto kantiano de legalidad (Habermas, 1994, p. 46). El derecho reserva al individuo dos alternativas de acción. Por un lado, el individuo posee la capacidad 
de actuar según su libertad subjetiva de acción cuando cumple la ley por causa de la coacción que es inherente al derecho; por otro, el derecho puede ser seguido por deber, cuando el ciudadano da su consentimiento a la ley en la medida en que es su propio autor.

Es necesario aclarar la interpretación de Habermas, porque, como es sabido, Kant introduce su célebre distinción entre derecho y ética basándose en el criterio subjetivo de la motivación de la acción, por tanto, el derecho no exige del individuo la acción por deber, sino tan solo conforme al deber. A pesar de que Habermas no hace referencia explícita a tal distinción al presentar el concepto kantiano de legalidad, no cabe ninguna duda de que la conoce; sin embargo, para simplificar su argumentación, prefiere no involucrarse en los matices de la filosofía kantiana del derecho, porque, al fin y al cabo, una ley jurídica también puede ser seguida por deber, si bien Kant afirma que en tal caso el sujeto la sigue éticamente, y no jurídicamente.

Habermas reconstruye el concepto kantiano de legalidad según la teoría de la acción comunicativa (Habermas, 1994, p. 47). Empieza por los derechos subjetivos, porque, desde Hobbes, las reglas del derecho privado burgués son el prototipo de los derechos en general. El doble aspecto de la validez del derecho: la facticidad resultante de la imposición del derecho positivo, expresada en la coerción, y la validez proveniente de las condiciones de producción del derecho legítimo, manifiesta en la libertad, posibilitan a los actores papeles sociales distintos. Por un lado, los actores pueden actuar según la facticidad del derecho en la medida en que adquiere vigencia social. Como afirmó Kelsen, el derecho posee una normatividad empírica, es decir, aparece para los actores como una obligación por el sencillo hecho de que su violación puede sancionarse por el derecho positivo. El carácter deontológico del derecho proveniente de su capacidad de coerción puede constituirse en el móvil de la acción de los actores sociales. El derecho subjetivo moderno no puede obligar a nadie a actuar coercitivamente por convicción en relación con las normas jurídicas. La modernidad, con la separación entre esfera pública y privada, la cual empieza, según Weber, con la doctrina luterana de la privatización de la fe, desplaza la motivación al ámbito de autonomía del sujeto (Habermas, 1994, p. 47).

Sin embargo, el derecho subjetivo puede ser seguido por actores movidos por la convicción de la legitimidad de la ley jurídica. La legitimidad del derecho se revela en la concepción de que las normas jurídicas justas son válidas, incluso, si no están contempladas en ningún código vigente o no son seguidas, de hecho, por nadie. La asociación del derecho con la metafísica y la religión en las sociedades 
convencionales prestó al derecho un significado de legitimidad que provenía de la objetividad de los valores o de la voluntad de Dios que permitía a los actores conectar su voluntad privada con la norma. Las sociedades posconvencionales ya no pueden servirse de la fundamentación metafísica o teológica realista de los valores, porque las sociedades se tornaron pluralistas, con múltiples concepciones de vida buena en función de los cambios en la eticidad tradicional, por consiguiente, no es ya aceptable la idea de una unidad en torno a los valores objetivos, que tendrían que valer incondicionalmente para todos. Sin embargo, para Habermas, el derecho moderno continúa sosteniendo una validez incondicional proveniente de los procedimientos democráticos de producción legislativa del derecho y del control judicial de la aplicación (Habermas, 1994, p. 49). Pero, sea como resultado de valores válidos objetivamente o del carácter democrático del procedimiento político, los actores tienen a su disposición la capacidad de actuar por convicción, y conectar su voluntad con la idea del derecho legítimo.

Habermas denomina tensión interna del derecho la doble validez del derecho, la cual se manifiesta en la doble característica del deber jurídico (Habermas, 1994, p. 52). Por un lado, el deber jurídico se constituye empíricamente en un deber por su vigencia social garantizada por el Estado de derecho; por otro, el deber jurídico posee el sentido de validez o de justicia que continúa obligando, incluso, en el caso de que no tenga ninguna vigencia social.

Los derechos subjetivos dependen naturalmente de ser complementados con derechos de otros tipos: los derechos de participación y comunicación política, para no hablar de derechos sociales que puedan garantizar por lo menos el mínimo necesario de condiciones reales de uso de los propios derechos. El derecho objetivo, por su parte, posee la misma estructura que cualquier derecho y también dispone de la doble validez de los derechos subjetivos, es decir, la coerción fáctica y la validez dimanante de la legitimidad, por consiguiente, tienen que permitir a los actores las dos formas de acción según el autointerés racional o la convicción motivada (Habermas, 1994, pp. 163-164). Igual que en el papel de destinatario del derecho que tiene derechos subjetivos, el ciudadano tampoco puede ser obligado a cumplir el derecho por deber en la función de autor del derecho. La coerción puede solo afectar el comportamiento del ciudadano, pero no su motivación. Así pues, el ciudadano tiene la libertad subjetiva de acción de rehusar su compromiso motivacional con leyes que son resultado del proceso legislativo, incluso, cuando este es democrático y legítimo. Sea en las democracias representativas modernas o en una república con participación directa de los ciudadanos, los autores pueden rehusar aceptar la justicia de las leyes, como ocurre con las minorías derrotadas en el proceso político. Las minorías tienen que seguir 
la ley por imposición, pero no pueden ser obligadas jurídicamente a seguirlas por convicción; además, las democracias modernas tienen que dejar la posibilidad de que tales minorías puedan continuar manifestando su punto de vista e intentar modificar la opinión y la decisión de la mayoría en otra sesión parlamentaria. Sin embargo, la naturaleza del proceso político permite, por otro lado, la virtud ciudadana de actuar por respeto a la ley (Habermas, 1994, p. 164). Eso no ocurre tan solo por medio del proceso democrático auténtico, ya que también los gobiernos autoritarios, o en especial ellos, demandan de los ciudadanos el ensanchamiento de las convicciones con las decisiones políticas. Por supuesto que hay una notable diferencia entre seguir una ley por convicción racional y seguir la ley por convicción manipulada, pero en los dos casos se exige más de los ciudadanos que el simple actuar según la libertad subjetiva de acción.

Además de la tensión interna resultante de la doble validez del derecho: la coerción fáctica y la legitimidad, hay otra tensión vinculada con el derecho que Habermas denomina tensión externa. Es, justamente, la que surge de la interpretación del concepto de derecho según la teoría de la sociedad (Habermas, 1994, p. 52). Al fin y al cabo, el derecho es un sistema social, por tanto, responsable de coordinar la acción, en concurrencia con otros sistemas sociales, en especial el mercado y la política, igualmente involucrados en la regulación de la acción.

En función de la doble validez del derecho, expresa en la tensión interna entre facticidad y validez, el derecho es adecuado para las condiciones modernas provenientes de la autonomización de los sistemas sociales y de la racionalidad estratégica. Los actores involucrados en el mercado y la política poseen la capacidad de actuar según el egoísmo racional y el autointerés. Pero el derecho también puede ser seguido a través de la racionalidad estratégica en la medida en que deja a los actores la posibilidad de actuar según su libertad subjetiva de acción. De este modo, el derecho, como sistema especializado, presenta la misma lógica de los demás sistemas y está en condiciones de hacerse comprender por ellos (Habermas, 1994, pp. 58-59). El lenguaje del derecho le hizo un sistema eficiente de regulación del mercado en caso de lesión de los intereses recíprocos o de protección de los grupos desaventajados; pero también de la política, pues el derecho posibilita el control de la política en situaciones de amenaza a las libertades individuales o a derechos colectivos.

No obstante, la legitimidad del derecho se relaciona con la acción comunicativa y la solidaridad del mundo de la vida. El derecho moderno es un sistema especializado que surge de la evolución de las prácticas comunicativas del mundo de la vida. Con la modernización social, la acción comunicativa, que es desempeñada 
naturalmente en el mundo de la vida, se torna reflexiva, y pasa a ser realizada en los discursos. Los discursos son formas de vida especiales porque las únicas acciones permitidas en ellos son las argumentaciones racionales. La acción comunicativa en el mundo de la vida es una amalgama de argumentaciones y otras acciones, pero la modernización permitió la autonomización de las argumentaciones en instituciones específicas. Igual que la comunidad científica, los parlamentos e, incluso, los tribunales, donde se produce y aplica el derecho, son también instituciones donde solo valen los argumentos. Pero, en tal circunstancia, el sistema jurídico es una forma reflexiva y especializada en la argumentación racional de la acción comunicativa del mundo de la vida. Eso implica que el derecho tiene que mantener la búsqueda cooperativa de las pretensiones de validez del mundo de la vida. Los parlamentos que producen el derecho no pueden ser solo el lugar de expresión del conflicto de intereses, sino también de la búsqueda del entendimiento. Del mismo modo, a pesar de que los tribunales no están orientados hacia el entendimiento, dado que los intereses de las partes están enfrentados, lo que se manifiesta en el papel del fiscal y del abogado, de la acusación y la defensa; en la perspectiva del juez, este conflicto de argumentos entre las partes tiene que resultar en informaciones indispensables para comprender exactamente los hechos y permitir la aplicación justa y neutral de la ley. El derecho se nutre de la solidaridad del mundo de la vida y eso se refleja en la otra forma de validez del derecho, o su legitimidad, que permite a los actores seguir el derecho por convicción y no solo por coacción (Habermas, 1994, pp. 58-59). Eso implica que el derecho también comprende el lenguaje comunicativo del mundo de la vida y su intervención sobre él no siempre significa una intromisión sistémica o colonización.

A causa de la tensión interna entre facticidad y validez, el sistema jurídico dispone de una capacidad de comunicación con todos los elementos de una sociedad moderna fragmentada. Además, permite la comunicación entre los sistemas entre sí o con el mundo de la vida. Por último, el derecho posibilita la preservación del mundo de la vida contra la intervención del mercado, el esclarecimiento del mundo de la vida contra sus propios autoengańos, lleva a una forma de vida elegida con consciencia y, por fin, sirve como conductor de las relaciones de entendimiento y solidaridad del mundo de la vida hacia los sistemas sociales. El sistema jurídico desempeña el papel de intermediario entre los sistemas y el mundo de la vida, y eso ofrece a Habermas la condición para explicar los problemas de integración social en sociedades posconvencionales (Habermas, 1994, p. 108). 


\section{Conclusiones}

La estructura social de la sociedad posconvencional resultó en una autonomía jurídica dividida en dos piezas: la autonomía privada, que permite a los agentes sociales utilizar la libertad subjetiva de acción, en que pueden elegir los motivos para seguir la norma, que puede ser tanto el autointerés, o el temor de la coacción, o aun su concepción particular de bien, y la autonomía pública, que exige el uso de libertad comunicativa, según la cual los agentes buscan el entendimiento mutuo (Habermas, 1992, p. 52).

Esa duplicación de la autonomía jurídica en la sociedad complexa explica la tensión interna entre facticidad y validez en el derecho. En el nivel de la norma jurídica, los destinatarios del derecho pueden usar su autonomía privada y obedecerla por causa de su vigencia como un hecho social que se impone coercitivamente o pueden considerar su autonomía pública cuando son motivados por la convicción racional oriunda de la aceptación de su legitimidad.

Por eso, el derecho moderno puede cumplir la función de integración social en la sociedad complexa y realizar la mediación entre el mundo de la vida y los sistemas funcionales, una vez que los agentes guiados por la racionalidad comunicativa del mundo de la vida siguen las normas por respeto a su validez, mientras los agentes, regidos por la racionalidad estratégica de los sistemas, calculan la relación costo-beneficio de su cumplimiento como un hecho social.

En este sentido, Habermas cambia su punto de vista sobre el sistema jurídico, pues, en Teoría de la acción comunicativa, de la década de 1980, consideró que el derecho era un sistema social que, junto con la economía y la política, también colonizaba el mundo de la vida, pero pensó que la racionalidad comunicativa del propio mundo de vida podría realizar la integración social, porque el lenguaje ordinario del mundo de la vida funciona como último metalenguaje de los lenguajes formales de los sistemas sociales. Sin embargo, en Facticidad y validez, piensa que el derecho puede ser responsable de la integración social, pues opera como traductor universal entre los diferentes lenguajes que se emplean en el mundo de la vida y los sistemas sociales. La integración social en las sociedades complejas es posible porque el derecho sirve como traductor universal, justamente, porque posee una doble forma de validez, pues el agente puede actuar tanto según la racionalidad comunicativa del mundo de la vida como según la racionalidad estratégica de los sistemas sociales. 


\section{Referencias}

Baxter, H. (2002a). Habermas's discourse theory of law and democracy. Buffalo Law Review, 50, 205-340.

Baxter, H. (2002b). System and lifeworld in Habermas's theory of law. Cardozo Law Review, 23, 473-615.

Baynes, K. (1992). The normative grounds of social criticism: Kant, Rawls and Habermas. SUNY.

Baynes, K. (1995). Democracy and the Rechtsstaats: Habermas's Faktizität und Geltung. En S. White (ed.), Cambridge companion to Habermas (pp. 201-32). Cambridge University Press.

Bohman, J. (1994). Complexity, pluralism, and the constitutional state: On Habermas's Faktizität und Geltung. Law and Society Review, 28(4), 897-930.

Cohen, J. L. y Arato, A. (1997). Civil society and political theory. Massachusetts Institute of Technology.

Cronin, C. P. (1993). Translator's introduction. En J. Habermas, Justification and application: Remarks on Discourse Ethics (pp. XI-XXXI). Massachusetts Institute of Technology.

Gimbernat, J. A. (ed.) (1997). La filosofía moral y política de Jürgen Habermas. Biblioteca Nueva.

Habermas, J. (1973). Technik und Wissenschaft als "Ideologie". Suhrkamp Verlag.

Habermas, J. (1992). Erläuterungen zur Diskursethik. Suhrkamp Verlag.

Habermas, J. (1994). Faktizität und Geltung: Beitrage zur Diskurstheorie des Rechts und des demokratischen Rechtsstaats. Suhrkamp Verlag.

Habermas, J. (1995). Theorie des Kommunikativen Handelns. Suhrkamp Verlag.

Habermas, J. (1996). Moralbewußtsein und Kommunikatives Handeln. Suhrkamp Verlag.

Habermas, J. (1997). Die Einbeziehung des Anderen: Studien zur politischen Theorie. Suhrkamp Verlag.

Jiménez Redondo, M. (1998). Introducción. En J. Habermas, Facticidad y validez (pp. 9-55). Trotta.

Rehg, W. (1996). Translator's introduction. En J. Habermas, Between facts and norms: Contributions to a discouse theory to law and democracy (pp. IX-XXXVII). Massachusetts Institute of Technology.

Rosenfeld, M. y Arato, A. (ed.) (1998). Habermas on law and democracy: Critical exchanges. University of California Press.

Vallespín, F. (1998). Introducción. En J. Habermas y J. Rawls, Debate sobre el liberalismo político (pp. 9-37). Paidós.

Velasco, J. C. (1999). Introducción. En J. Habermas, La inclusión del otro (pp. 11-22). Paidós.

Velasco, J. C. (2000). La teoría discursiva del derecho: Sistema jurídico y democracia en Habermas. Centro de Estudios Políticos y Constitucionales. 